\title{
Cutaneous manifestation of chronic lymphocytic leukemia: a case report and a literature review
}

\author{
Łukasz Szukalski ${ }^{1}$, Martyna Sysakiewicz-Buda², Kaja Męcińska-Jundziłł ${ }^{3}$ Agnieszka Białecka³, Jarosław Czyż ${ }^{1}$, \\ Rafał Czajkowski ${ }^{3}$
}

${ }^{1}$ Department of Haematology, Collegium Medicum in Bydgoszcz, Nicolaus Copernicus University in Torun, Poland ${ }^{2}$ Department of Haematology, Nicolaus Copernicus Hospital, Torun, Poland

${ }^{3}$ Chair of Dermatology, Sexually Transmitted Diseases and Immunodermatology, Faculty of Medicine, Nicolaus Copernicus University in Torun, Poland

Adv Dermatol Allergol 2019; XXXVI (6): 778-780

DOI: https://doi.org/10.5114/ada.2019.91427

B-cell chronic lymphocytic leukemia (CLL) is the most prevalent type of leukemia among people aged 65 and more [1]. It usually develops in a predictable way, initially manifesting itself in peripheral blood and subsequently entering also extramedullary locations: the lymph nodes, the spleen, and the liver, described in detail by Rai et al., and became the foundation of the classical prognostic system [2]. Various skin lesions are relatively frequent among people suffering from CLL but a real lesion of the skin by leukemic cells called leukemia cutis (LC) is a rare manifestation and may be associated with a more aggressive course of the disease [3, 4].

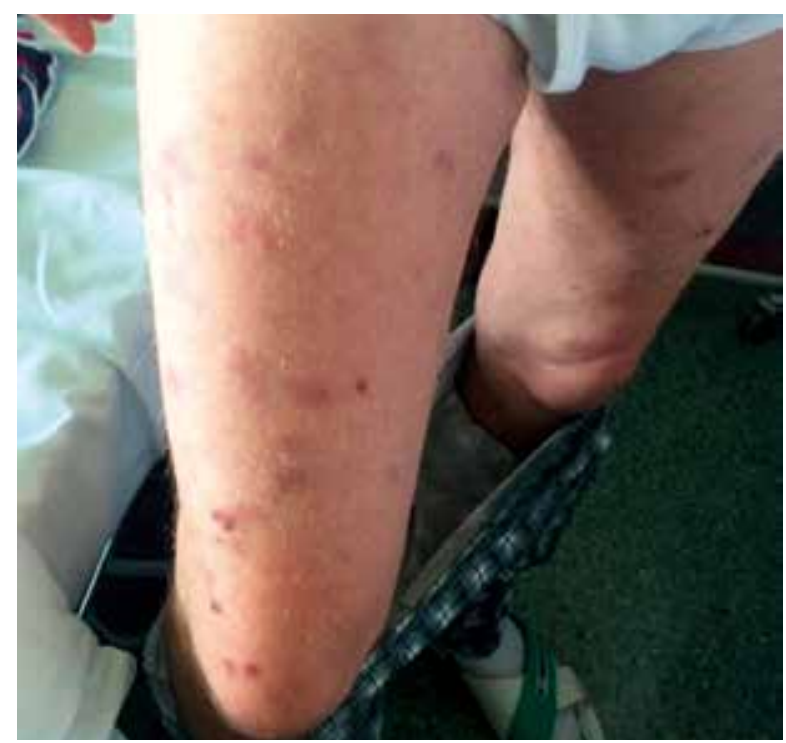

Figure 1. Cutaneous lesions on thighs of a patient with chronic lymphocytic leukemia before starting chemotherapy
In this paper we present a patient with a histopathologically confirmed case of infiltration of the skin by chronic lymphocytic leukemia lymphocytes. The occurrence of skin lesions correlated with the disease's progression. In the case report we both refer to the results of the treatment and present the literature pertaining to the topic introduced above.

Course of the disease: In 2014, a 68-year-old man went through a routine check-up which showed an elevated lymphocyte count in peripheral blood. At that time, the patient showed no general symptoms, such as night sweats, raised temperature or loss of weight. Physical examination did not show enlarged lymph nodes, liver or spleen. Laboratory tests established the white blood cell count of $26.80 \mathrm{G} / \mathrm{l}$, haemoglobin concentration of $15.6 \mathrm{~g} / \mathrm{dl}$ and platelet count of $220 \mathrm{G} / \mathrm{l}$, small lymphocytes were dominant in the blood smear. Between 2014 and 2017 the patient remained under observation without getting any treatment.

In March 2017, the patient observed a single skin lesion on his lower abdomen, accompanied by redness and mild itching. About a month later, he noticed increased night sweating, without fever or loss of weight. Physical examination showed enlarged lymph nodes and spleen (ca. $2 \mathrm{~cm}$ below the left rib arch) but no palpable enlargement of the liver. In the following weeks, there was a progression of the skin lesions in the form of numerous red and blue-purple lumps and bumps on the skin on the patient's arms, back, lower abdomen and thighs (Figure 1). At that time, laboratory tests showed an elevated white blood cell count of $142.61 \mathrm{G} / \mathrm{l}$, haemoglobin concentration was $14.3 \mathrm{~g} / \mathrm{dl}$ while platelet count was $167 \mathrm{G} / \mathrm{l}$. The blood smear showed $92 \%$ of small lymphocytes, typical of the classical form of chronic lymphocytic leukemia.

Address for correspondence: Łukasz Szukalski MD, Department of Haematology, Collegium Medicum, Nicolaus Copernicus University, 75 Ujejskiego St, 85-001 Bydgoszcz, Poland, phone: +48 729104 471, e-mail: lukaszszukalski@gmail.com Received: 10.10 .2018 , accepted: 2.11.2018. 
Histopathological evaluation of the skin lesions: immunohistochemistry tests of skin biopsies taken from the left arm area and back showed perivascular lesions of small B lymphocytes CD20(+), CD23(+), and some CD5(+) typical of the classical form of CLL. Additionally, CD3+ inflammatory T-cells were present in the dermis. On the whole, it was confirmed that an infiltration of the skin occurred due to CLL.

Treatment: the occurrence of general symptoms led to the decision to start treatment. Because of the relatively young age of the patient and the lack of relevant accompanying diseases, the FCR chemotherapy regimen was employed (fludarabine, cyclophosphamide, rituximab). The patient received a total of six cycles of the FCR regimen. After the first course of chemotherapy a substantial regression of skin lesions and the previously enlarged lymph nodes was observed, with continuing liver and spleen enlargement. A total clearance of skin lesions was observed after the third cycle of chemotherapy.

As a result of the employed treatment, a hematologic remission and total clearance of the skin lesion was achieved. The patient is under observation as an outpatient.

Skin lesions observed in patients suffering from leukemia can be divided, depending on the clinical and histopathological features, into specific and nonspecific. Non-specific lesions are either connected with leukemia and result from haematopoiesis disorders or a manifestation of cutaneous paraneoplastic syndrome or a consequence of the side effects of the medication. They include purpura petechiae resulting from thrombocytopenia, severe skin infections such as disseminated shingles, furunculosis or fungal abscesses caused by agranulocytosis or drug-related allergic reactions of the skin [5]. Specific skin lesions are those caused by leukemic cells, irrespective of their morphology called leukemia cutis (LC) [5-7].

Skin lesions occurring in the course of chronic lymphocytic leukemia are widely described in the literature [3-5, 7-10]. They are observed in as many as $25 \%$ of patients diagnosed with CLL $[4,11]$. It deserves a mention, however, that the great majority of lesions occurring are non-specific $[8,9]$. Specific lesions by leukemic cells of the leukemia cutis type are a rarity among the CLL skin lesions, their incidence being 4-27\% [6, 8, 10, 11].

Skin lesions of the leukemia cutis type do not always appear after leukemia is diagnosed but according to the literature, it is the case in $55-77 \%$ of LC cases and the average duration of CLL before LC occurs is 39 months as shown in the study described by Cerroni et al. [3]. Skin lesions and leukemia occur concurrently in $23-38 \%$ of cases. The appearance of specific leukemic lesions prior to leukemia diagnosis (months or even years before) is rare (7-16.7\%) and was named "aleukemic leukemia cutis" $[3,5,12]$.
No common locations of leukemia cutis lesions were established. The torso, limbs and head alike are subject to lesions with equal frequency $[3,13]$. LC is less common in palms and soles as well as the mucous membrane of the mouth cavity [12].

Skin lesions of the leukemia cutis type may be single or numerous and occupy a limited area or be disseminated [4]. The most frequently described clinical manifestation of LC are lumps and bumps of various size [12]. Less commonly, skin lesions may manifest themselves as erythematous lesions, petechiae, haemorrhoids, ulcerations, blisters and subungual lesions [3, 5, 6, 10, 12].

The histological picture of $L C$ varies depending on the course of the disease and does not necessarily correlate with the clinical picture. In a study conducted by Klco et al., it was established that in around a third of the patients with LC, a disseminated distribution pattern of the lesion, spanning the surface and deep layers of the dermis, occurred. Localised, perivascular or periadnexal growth patterns were also described $[3,14]$.

The molecular background of the invasion of leukemic cells into the skin has not yet been explained. Cho-Vega et al. presume that intercellular adhesion molecule 1 (ICAM-1) and lymphocyte function-associated antigen 1 (LFA-1) factors can play a substantial role in the pathogenesis of lesions in the course of B-CLL and that the lesion mechanism can be similar to that which skin cells employ to recruit T-lymphocytes [7].

The influence of ICAM-1 and LFA-1 levels on leukemic cell migration into the skin has been described previously [15].

What might be an important fact for explaining the pathogenesis is that cases of leukemia cutis lesions in the place of post-infection scars or other inflamed places are frequently described in the literature. In a publication by Cerroni et al., as many as 6 out of 42 patients with CLL had leukemia cutis type lesions in the place of scars after a herpes infection [3]. Such leukemic lesions in scars after herpes virus eruptions have been described by other authors as well [16]. Walther et al. described 6 cases of CLL in the course of which lesions appeared in the places where skin cancer developed, in four of squamous cell carcinoma cases and in two basal cell carcinomas [13]. Numerous other authors described tissues of the B-CLL phenotype present in skin cancer specimens being at the same time a primary or secondary manifestation of chronic lymphocytic leukemia $[9,17]$.

Such accumulation of Leukemia cutis lesions in inflammatory lesions, scars, or in skin neoplasms can be explained by the mechanism, which causes interleukin 1 (IL-1) released from damaged keratinocytes and tumor necrosis factor (TNF) present in skin cancer cells to activate ICAM-1 and lead to B-lymphocyte recruitment into the skin in a way similar to T-lymphocyte migration into the skin [18]. 
In the case of LC, differential diagnosis depends on the morphology, number and location of skin lesions. In the case of single skin lesions on the torso and limbs, depending on the morphology, lymphomas, Kaposi's sarcoma and both basal and squamous cell carcinoma must be taken into consideration. In the case of numerous lesions, they must be differentiated between drug eruptions, viral infections, syphilitic exanthema, pityriasis rosea, hypersensitivity vasculitis and autoimmune blistering diseases. In the case of lesions located on the face, seborrheic dermatitis and systemic lupus erythematosus must be taken into consideration in differential diagnosis as well [6].

It is not clear whether leukemia cutis type lesions influence the prognosis for patients with $\mathrm{B}-\mathrm{CLL}[3,10,12$, 13]. Some authors claim that the presence of skin lesions consisted of small lymphocytes can have prognostic importance $[3,13]$. Colburn et al. described 2 cases with the presence of lymphocytic skin lesions in the course of CLL correlating with a favourable prognosis [10]. Unfavourable prognosis was, however, also noticed in patients with leukemia cutis lesions in the course of Richter's syndrome and when leukemic lesions occurred after B-CLL diagnosis [3]. Su et al. have described death of 12 out of 16 patients with CLL on average 16 months after LC lesions developed, what have led the authors to assume that $L C$ is a very unfavourable prognostic factor [12].

\section{Acknowledgments}

The department in which the study was conducted and the work should be attributed to: Department of Haematology, Collegium Medicum in Bydgoszcz, Nicolaus Copernicus University in Toruń, Poland

\section{Conflict of interest}

The authors declare no conflict of interest.

\section{References}

1. Rai KR, Jain P. Chronic lymphocytic leukemia (CLL) - then and now. Am J Hematol 2016; 91: 330-40.

2. Rai KR, Sawitsky A, Cronkite EP, et al. Clinical staging of chronic lymphocytic leukemia. Blood 1975; 46: 219-34.

3. Cerroni L, Zenahlik P, Höfler G, et al. Specific cutaneous infiltrates of B-cell chronic lymphocytic leukemia: a clinicopathologic and prognostic study of 42 patients. Am J Surg Pathol 1996; 20: 1000-10

4. Watson KM, Mufti G, Salisbury JR, et al. Spectrum of clinical presentation, treatment and prognosis in a series of eight patients with leukaemia cutis. Clin Exp Dermatol 2006; 31: 218-21.

5. Ratnam KV, Kohr CJ, Su WP. Leukemia cutis. Dermatol Clin 1994; 12: 419-31.

6. Wagner G, Fenchel K, Back W, et al. Leukemia cutis - epidemiology, clinical presentation, and differential diagnoses. J Dtsch Dermatol Ges 2012; 10: 27-36.
7. Cho-Vega JH, Medeiros LJ, Prieto VG, Vega F. Leukemia cutis. Am J Clin Pathol 2008; 129: 130-42.

8. Agnew KL, Ruchlemer R, Catovsky D, et al. Cutaneous findings in chronic lymphocytic leukaemia. Br I Dermatol 2004; 150: 1129-35.

9. Padgett JK, Parlette HL, English JC. A diagnosis of chronic lymphocytic leukemia prompted by cutaneous lymphocytic infiltrates present in Mohs micrographic surgery frozen sections. Dermatol Surg 2003; 29: 769-71.

10. Colburn DE, Welch MA, Giles FJ. Skin infiltration with chronic lymphocytic leukemia is consistent with a good prognosis. Hematology 2002; 7: 187-8.

11. Greenwood R, Barker DJ, Parapia L, et al. Clinical and immunohistological characterization of cutaneous lesions in chronic lymphocytic leukaemia. Br J Dermatol 1985; 113: 447-53.

12. Su WPD, Buechner SA, Chin-Yang Li. Clinicopathologic correlations in leukemia cutis. J Am Acad Dermatol 1984; 11: 121-8.

13. Walther BS, Gibbons G, Chan EF, et al. Leukemia cutis (involving chronic lymphocytic leukemia) within excisional specimens: a series of 6 cases. Am J Dermatopathol 2009; 31: 162-5.

14. Klco JM, Welch JS, Nguyen TT, et al. State of the art in myeloid sarcoma. Int J Labor Hematol 2011; 33: 555-65.

15. Uccini S, Ruco LP, Monardo F, et al. Molecular mechanisms involved in intraepithelial lymphocyte migration: a comparative study in skin and tonsil. J Pathol 1993; 169: 413-9.

16. Zimmer M, Bornkessel A, Hahnfeld S, Weyers W. "Specific" cutaneous infiltrate of B-cell chronic lymphocytic leukemia at the site of a florid herpes simplex infection. J Cutan Pathol 2005; 32: 581-4.

17. Albregts T, Orengo I, Salache $S$, et al. Squamous cell carcinoma in a patient with chronic lymphocytic leukemia. Dermatol Surg 1998; 24: 269-72.

18. Yang L, Froio RM, Sciuto TE, et al. ICAM-1 regulates neutrophil adhesion and transcellular migration of TNF-alphaactivated vascular endothelium under flow. Blood 2005; 106: 584-92. 